\title{
Psicooncología
}

ISSN: 1696-7240

http://dx.doi.org/10.5209/PSIC.57087

\section{Reconstrucción mamaria y calidad de vida}

\author{
Jaume Masià̀,*; Elena Rodríguez Bauzà
}

Recibido: 1 de septiembre de 2015 / Aceptado: 25 de agosto de 2017

Resumen. Introducción: la calidad de vida es un concepto fuertemente condicionado por la integridad física e imagen corporal de un individuo. La reconstrucción mamaria se ha convertido en una opción idónea para las mujeres diagnosticadas de cáncer de mama. Es un paso esencial en la recuperación de las secuelas físicas y psíquicas que produce el tratamiento del cáncer de mama en la mujer, y debe ser analizada de forma individual. Procedimientos reconstructivos: existen diversas técnicas reconstructivas, que deben satisfacer el objetivo estético de recuperar el contorno de la mama, así como una mejoría funcional para la mujer con el fin de mejorar su calidad de vida. La reconstrucción puede realizarse mediante implantes, mediante tejido del propio cuerpo o reconstrucción mediante técnicas mixtas. Por sus características, el colgajo DIEP se ha convertido en la técnica quirúrgica más fisiológica y de elección para la reconstrucción mamaria. Discusión: las pacientes tienen derecho a una valoración exhaustiva y rigurosa, por parte de su cirujano plástico, de la técnica más indicada para su intervención de reconstrucción mamaria. El hecho de que sólo el hecho de que sólo el $30 \%$ de las mujeres que se someten a una mastectomía lleven a cabo la reconstrucción, se debe principalmente a una falta de información, afectando claramente a la calidad de vida de las pacientes. Conclusión: la reconstrucción mamaria no debería considerarse un paso posterior, si no una parte imprescindible para un tratamiento integral.

Palabras clave: Calidad de vida; reconstrucción mamaria; colgajo DIEP; prótesis mamaria; colgajo dorsal ancho; expansor tisular; cáncer de mama.

\section{[en] Breast reconstruction and quality of life}

Abstract. Introduction: Quality of life is a concept strongly conditioned by the physical integrity and individual body image. Breast reconstruction has become an ideal choice for women diagnosed with breast cancer. It is an essential step and should be analysed individually. Reconstructive procedures: Reconstruction can be performed through implants and through autologous tissue and can be immediate or delayed. The DIEP flap has become the gold standard in breast reconstruction. Discussion: Patients should be advised by their plastic surgeon about the most appropriate technique for their breast reconstruction. The fact that only $30 \%$ of women who undergo mastectomy carry out reconstruction is mainly due to a lack of information. Conclusion: Breast reconstruction should not be considered a posterior step of breast cancer treatment. Breast reconstruction should be considered an essential part for an integral treatment.

Keywords: Quality of life; Breast reconstruction; DIEP flap; breast prostheses; wide dorsal muscle flap; tissue expander, breast cancer.

1 Department of Plastic Surgery, Hospital de la Santa Creu i Sant Pau. Universitat Autonòma de Barcelona Department of Plastic Surgery, Hospital de la Santa Creu i Sant Pau. Universitat Autonòma de Barcelona.

E-mail: jmasia@santpau.cat

2 Elena Rodríguez-Bauzà. Department of Plastic Surgery, Hospital de la Santa Creu i Sant Pau. Universitat Autonòma de Barcelona.

E-mail: erodriguezb@santpau.cat

* Dirección de correspondencia: Jaume Masià. Department of Plastic Surgery, Hospital de la Santa Creu i Sant Pau. Universitat Autonòma de Barcelona. Sant Quintí, 89, 08026, Barcelona, Spain. E-mail: jmasia@santpau.cat 
Sumario. 1. Introducción 2. Procedimientos reconstructivos 3. Linfedema 4. Discusión 5. Conclusión 6. Referencias bibliográficas.

Cómo citar: Masià J, Rodríguez Bauzà E. Reconstrucción mamaria y calidad de vida. Psicooncología 2017;14:295-306. Doi: 10.5209/PSIC.57087.

\section{Introducción}

El cáncer de mama afecta aproximadamente a 1 de cada 8 mujeres, siendo el tumor más frecuente en la población femenina tanto en países desarrollados como en aquellos en vías de desarrollo ${ }^{(1)}$. El interés por la calidad de vida en las pacientes afectas de cáncer de mama se ha incrementado en los últimos años y es objeto de considerable importancia en la evaluación global del resultado del tratamiento. La mejora en la detección y tratamiento precoz ha llevado a una mayor supervivencia en estas pacientes, cada vez más preocupadas por los efectos producidos a largo plazo de los distintos tratamientos.

La calidad de vida es un concepto fuertemente condicionado por la integridad física e imagen corporal de un individuo. Por esta razón, es esperable que el cáncer de mama pueda tener efectos graves en la calidad de vida de las mujeres afectas. Diversos estudios sobre mujeres que se someten a una mastectomía encuentran correlaciones entre el resultado estético y el nivel de depresión y ansiedad ${ }^{(2-6)}$. La reconstrucción mamaria se ha convertido en una opción idónea para las mujeres diagnosticadas de cáncer de mama, y en los últimos años, ha aumentado el número de pacientes que solicitan una reconstrucción mamaria después de una mastectomía ${ }^{(7-8)}$.

El estudio nacional de la Sociedad Española de Cirugía Plástica, Reparadora y Estética (SECPRE) sobre la calidad de tratamiento integral a las mujeres afectadas por cáncer de mama, sugiere que en torno al $30 \%$ de las mujeres afectas de cáncer de mama se someten a reconstrucción. De éstas, seis de cada diez la realizan de forma inmediata mientras que las otras cuatro la realizan de forma diferida. Es importante remarcar que un 21,3\% manifiesta que la información que se les facilitó sobre las posibilidades de reconstrucción fue insuficiente ${ }^{(9)}$. Este dato pone de manifiesto que no todas las mujeres tienen información sobre las posibilidades de reconstrucción antes de enfrentarse a un proceso de mastectomía y el hecho de que sólo el 30\% de las mujeres lleven a cabo la reconstrucción puede deberse, en parte, a una falta de información ${ }^{(10)}$.

El perfeccionamiento de las técnicas de reconstrucción mediante tejido autólogo, así como las mejoras en el diseño, textura y material de las prótesis mamarias, y el desarrollo de nuevos sustitutos de tejidos, han inducido avances notables en la reconstrucción mamaria ${ }^{(6,11,12)}$. De esta manera, el enfoque de la reconstrucción mamaria debe adaptarse para alcanzar un balance adecuado entre la minimización del riesgo de recurrencia y proporcionar el mejor resultado estético.

La reconstrucción mamaria debe valorarse como algo más que el hecho de reconstruir un pecho en el contexto de una mastectomía. Es un paso esencial en la recuperación de las secuelas físicas y psíquicas que produce el tratamiento del 
cáncer de mama en la mujer, y debe ser analizada de forma individual. Cada paciente presenta una historia médica diferente con tratamientos diferenciados, tiene unas características físicas que la hacen única y vive en un entorno afectivo personal que puede condicionar la elección de nuestra técnica. Por tanto, la única forma de conseguir una buena reconstrucción mamaria empieza con un estudio adecuado de la enfermedad y sobretodo de la mujer que la padece.

Nuestro objetivo en este artículo es definir los principales tipos de procedimientos reconstructivos en pacientes que se someten a una mastectomía, así como el análisis de la afectación de los mismos en la calidad de vida de nuestras pacientes.

\section{Procedimientos reconstructivos}

Existen diversas técnicas reconstructivas, que deben satisfacer el objetivo estético de recuperar el contorno de la mama, así como una mejoría funcional, con el fin de mejorar su calidad de vida ${ }^{(13,14)}$.

La reconstrucción puede realizarse mediante implantes (reconstrucción aloplástica), mediante tejido del propio cuerpo (reconstrucción con tejido autólogo) o reconstrucción mediante técnicas mixtas (colocación de implante + tejido autólogo). Todas estas técnicas tienen una serie de indicaciones que se analizarán a continuación, siendo más adecuadas unas u otras dependiendo de las características físicas y psíquicas de cada paciente.

Las técnicas que han ganado más popularidad en los últimos años son las que utilizan tejido de la propia paciente para recrear la mama, puesto que ofrece resultados muy similares a los de una mama natural.

No obstante, en algunas pacientes la reconstrucción utilizando implantes mamarios es más apropiada.

\subsection{Reconstrucción con implantes mamarios}

\section{- Implantes tipo expansor}

La reconstrucción en dos etapas es el tipo más común en el caso de reconstrucción con implantes mamarios. En la primera etapa, se coloca un expansor tisular, el cual es similar a un globo (prótesis vacia), debajo de la piel y de los músculos del tórax (principalmente músculo pectoral mayor). A través de una válvula diminuta debajo de la piel y el músculo, el cirujano plástico inyecta una solución de suero salino a intérvalos regulares para llenar el expansor con el paso del tiempo hasta conseguir el volumen deseado (tiempo medio de alrededor de 2 a 6 meses en función el volumen a conseguir y de las características de expansión de la piel).

Este proceso permite que la mayoría de la piel del tórax se distienda sobre el expansor, y cree así una "nueva" cobertura para la mama a reconstruir. En la mayoría de los casos, cuando la piel se ha distendido lo suficiente, el expansor se retira quirúrgicamente y se reemplaza por un implante de mama permanente (segunda etapa). 


\section{- Implantes directos}

La colocación de un implante directo está indicada en reconstrucciones mamarias inmediatas en aquellas mujeres que preferiblemente no vayan a recibir radioterapia y que por el tipo de tumor, se someten a una mastectomía conservadora de piel. Éstos pueden aplicarse en mujeres con pecho poco voluminoso y que tengan suficiente piel y una estructura torácica adecuada para acoger un implante directo durante la reconstrucción, y también en pechos voluminosos siguiendo un patrón de mastectomía de reducción. Este tipo de cirugía debe realizarse de forma muy cuidadosa, puesto que el colgajo de piel de la mastectomía tiene una irrigación pobre, y en el caso de no conservar correctamente el plexo subdérmico, se podría producir sufrimiento del colgajo cutáneo, con la posterior extrusión de la prótesis.

La aparición en los últimos años de mallas tanto biológicas como sintéticas que se colocan en el polo inferior de la mama o cubriendo completamente la prótesis han incrementado de forma considerable la indicación de este tipo de cirugía, permitiendo la reconstrucción con implantes en un solo tiempo. Aun así, la colocación de las mallas no esta exenta de complicaciones y sus indicaciones en cirugía reconstructiva son limitadas ${ }^{(15-16)}$.

La reconstrucción con implantes ofrece algunas ventajas como la brevedad de la intervención, la sencillez técnica y el hecho de que no produce nuevas cicatrices en zonas donantes. La reconstrucción con implantes puede ser una buena opción en pacientes que no hayan recibido radioterapia, en pacientes delgadas con poco tejido autólogo para recrear una nueva mama, o en pacientes, que por sus condiciones médicas o por sus propias preferencias, requieran una intervención más sencilla y corta.

Por otra parte, aunque la cirugía sea más corta, la paciente puede requerir un periodo más largo en el proceso de reconstrucción sobre todo si se realiza la reconstrucción con expansor tisular, necesitando de dos tiempos quirúrgicos.

Al ser los implantes elementos extraños al cuerpo humano, pueden precisar un reemplazo en el futuro, por lo que presenta una menor longevidad y estabilidad respecto a la reconstrucción con tejido autólogo.

Además, puede ser difícil conseguir una forma natural con respecto a la mama contralateral, por lo que este tipo de intervención suele recomendarse en pacientes con mamas pequeñas, que no tienden a caer mucho con el paso del tiempo; o bien si la paciente va a ser mastectomizada de forma bilateral, ya que es más fácil conseguir una adecuada simetría. Los implantes no presentan la misma evolución que la mama natural, por lo que con el paso de los años, el implante acaba creando una asimetría entre ambos pechos que a menudo precisa de cirugía plástica adicional.

No debe olvidarse, que en algunas mujeres se pueden producir complicaciones como la contractura capsular o la intolerancia del implante, sobretodo si han sido sometidas a radioterapia.

En resumen, la reconstrucción con implantes ha sido respaldada con frecuencia en las pacientes con mínimas zonas donantes o en aquellas que deseen minimizar la deformidad de las zonas donantes. Aunque el resultado es generalmente menos natural en aspecto y en sensación, que el que se puede lograr con una reconstrucción con tejido autólogo (además de la inestabilidad temporal a largo plazo de este tipo de reconstrucción), puede proporcionar resultados razonables en las pacientes adecuadamente seleccionadas (Figuras 1A, 1B y 1C). 


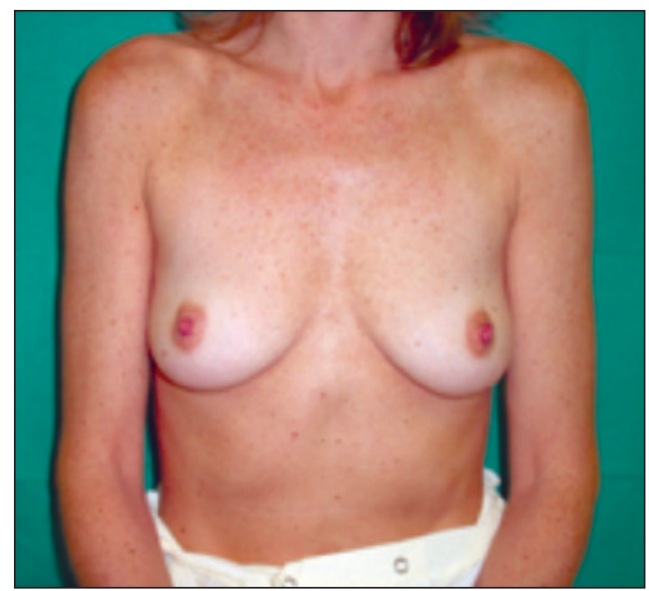

Figura 1A

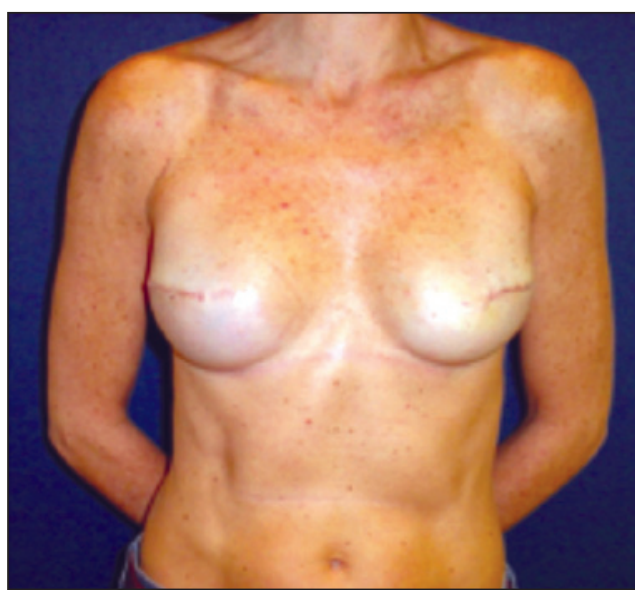

Figura 1B

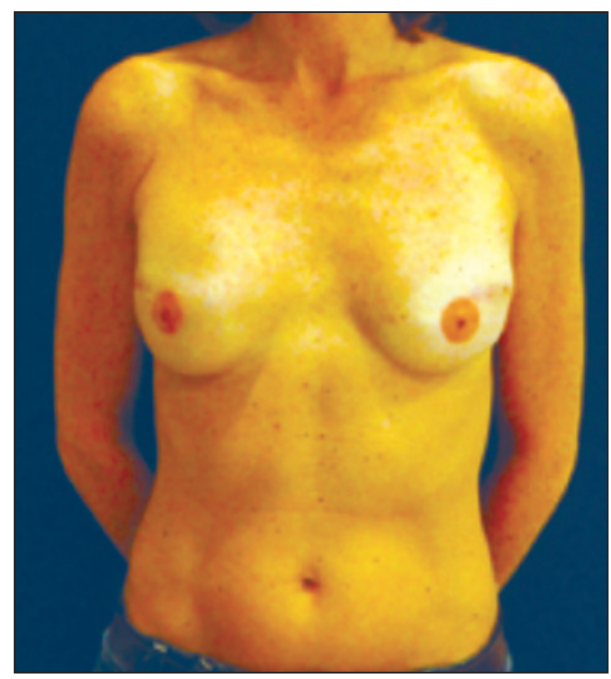

Figura $1 \mathrm{C}$

Figura 1A 1B y 1C. Mujer de 47 años que se sometió a mastectomía bilateral de reducción del riesgo y reconstrucción inmediata con implante mamario (implante mamario anatómico de 285 gr). La paciente rechazó la preservación del complejo areola del pezón. La figura 1A muestra las imágenes preoperatorias. La figura $1 \mathrm{~B}$ muestra el resultado postoperatorio a los 6 meses. La figura 1C muestra el resultado postoperatorio después de la reconstrucción de la areola del pezón al año postoperatorio.

\subsection{Reconstrucción con tejido autólogo}

en los últimos años, los resultados de reconstrucción mamaria obtenidos con tejido autólogo han demostrado ser excelentes presentando mayor estabilidad a largo plazo. El tejido del abdomen, por sus características, es considerado como la zona 
donante idónea. Nos proporciona, en la mayoría de los casos, el volumen suficiente para recrear una nueva mama, y las características inherentes de la piel abdominal (color y grosor) y de la grasa abdominal (consistencia) la convierten en el tejido más similar a un pecho natural.

\section{- Reconstrucción con colgajo latissimus dorsi}

Se trata de una de las técnicas más empleadas en las décadas de los ochenta y noventa, creada originalmente para ayudar a las mujeres que habían sufrido mastectomías radicales. Esta técnica consiste en extraer una cantidad significativa de piel y grasa de la espalda, así como parte del músculo dorsal ancho, y llevarla hasta el pecho a través de un túnel bajo la piel de la axila para reconstruir una mama con o sin necesidad de implantes. La operación deja una cicatriz de cierta longitud en la mitad de la espalda de donde se extrae el colgajo.

Aunque no es común, algunas mujeres pueden padecer debilidad en la espalda, el hombro o el brazo después de esta cirugía al utilizar el músculo dorsal ancho para la reconstrucción ${ }^{(17)}$.

La técnica del dorsal ancho, muy utilizada en los últimos 20 años, y con resultados muy seguros, conlleva un sacrificio muscular, las características de la piel de la espalda (piel más gruesa y de mayor pigmentación) no la definen como la más apropiada para sustituir a la de la mama y con mucha frecuencia precisan de la colocación de implantes para obtener suficiente proyección.

\section{- Colgajo DIEP (deep inferior epigastric perforator)}

El colgajo DIEP se ha convertido en la técnica "gold-standard" de la reconstrucción mamaria, ya que permite trasplantar únicamente piel y grasa de la zona inferior del abdomen, sin alterar la función muscular de la zona. La vascularización del tejido trasplantado se realiza mediante unos vasos llamados perforantes que provienen del sistema epigástrico inferior profundo.

La técnica DIEP requiere de un aprendizaje más largo que otros métodos de reconstrucción, precisando de un elevado grado de destreza para poder realizar la disección del colgajo con éxito, pero con equipos quirúrgicos experimentados es una técnica totalmente segura y que ofrece unos buenos resultados.

El tejido abdominal proporciona, en un gran número de casos, un volumen suficiente para la reconstrucción mamaria. Al poder prescindir del uso de implantes mamarios no se percibe la sensación de cuerpo extraño, ni los riesgos e inconvenientes que se derivan de su aplicación (contractura capsular, extrusión, recambios por desgaste ...).

La morbilidad de la zona donante es mínima. Al preservar la totalidad del músculo con su fascia, su inervación segmentaria y su circulación colateral, el riesgo de ocasionar debilidad de la pared abdominal es mínimo. De la misma forma al no haber un sacrificio funcional del músculo, la paciente mantiene integra su capacidad física. La recuperación postoperatoria más rápida y menos dolorosa que con otras técnicas que usan tejido autólogo y las dosis analgésicas postoperatorias son más bajas que con técnicas más agresivas (TRAM, latissimus dorsi ampliado ...). La técnica del colgajo TRAM se introdujo en la década de 1980 como la primera técnica que transfería tejidos del abdomen para reconstruir una mama. En su momento supuso un gran paso para mejorar las cirugías reconstructivas de la mama, aunque ha sido desplazado 
por las técnicas DIEP y el SIEA, ya que con estas técnicas únicamente se utiliza la piel y la grasa subcutánea, preservando la continuidad de las fibras musculares, la inervación motora del músculo y su circulación colateral. De esta forma se asegura la funcionalidad y la integridad de la pared abdominal.

En muchas ocasiones se puede ofrecer un beneficio estético al abdomen. La exéresis del tejido abdominal se realiza siguiendo los patrones de la dermolipectomía abdominal estética, intentando conseguir el mejor resultado posible, asociando si se precisa una plicatura abdominal o técnicas de remodelación de flancos.

Todas estas características nos llevan a definir el DIEP como la técnica quirúrgica más fisiológica y de elección para la reconstrucción mamaria (Ver la figura 2A y 2B).

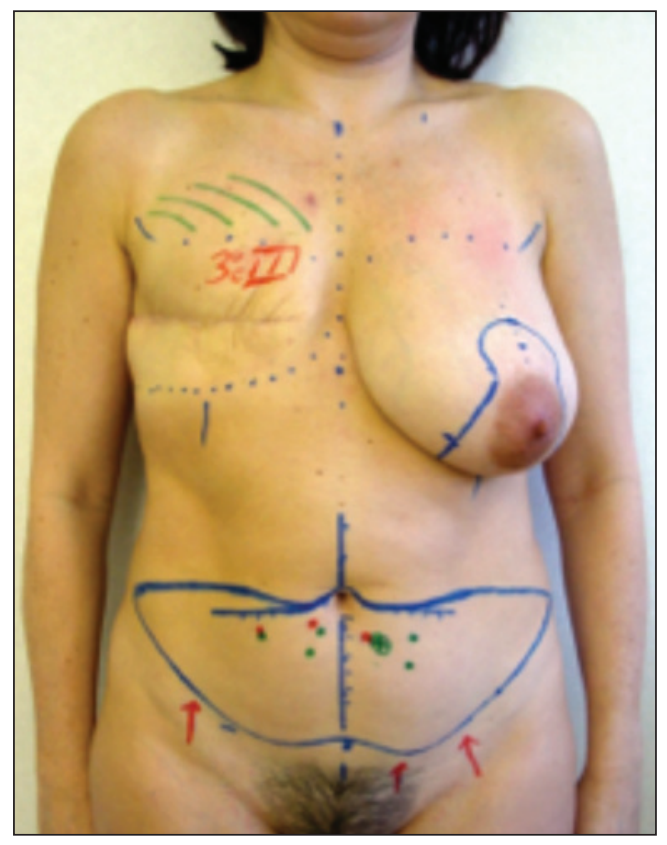

Figura 2A

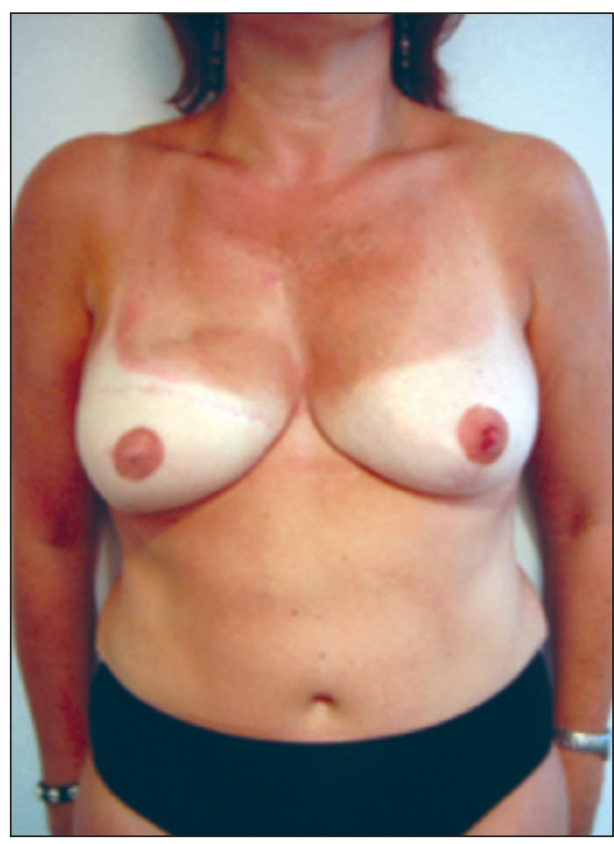

Figura 2B

Figuras 2A y 2B. Mujer de 58 años que se sometió a reconstrucción mamaria derecha diferida con colgajo DIEP y simetrización de mama izquierda. La figura 2A muestra el marcaje preoperatorio. La figura $2 \mathrm{~B}$ el resultado postoperatorio a los 9 meses.

Únicamente como inconvenientes destacaríamos que la técnica DIEP precisa de una curva de aprendizaje más larga que las técnicas convencionales y requiere de un esfuerzo y perseverancia mayor por parte del equipo quirúrgico.

En nuestra experiencia, aplicando de una forma clara y concisa este planteamiento terapéutico, la mayoría de pacientes optan por la reconstrucción con DIEP. Contraindicándola de forma absoluta, únicamente, en aquellas pacientes que por una alteración de la pared abdominal o por falta de exceso de tejido abdominal no se puede realizar. Como contraindicaciones relativas destacaríamos el tabaquismo activo y edad superior a 70 años. 


\section{- Colgajo siea (superficial inferior epigastric artery)}

El colgajo SIEA utiliza el mismo tejido que el colgajo DIEP para la reconstrucción mamaria con la diferencia de basar la vascularización del colgajo en los vasos epigástricos inferiores superficiales. Tiene la ventaja de ser la técnica menos invasiva y la que menos morbilidad ocasiona a la paciente al no tener que abrir la fascia abdominal para buscar los vasos del sistema profundo.

El único inconveniente es que no todas las pacientes disponen de un sistema superificial para poder realizar la reconstrucción mamaria con este colgajo (FIGURA $3 \mathrm{~A}$ y $3 \mathrm{~B})$.

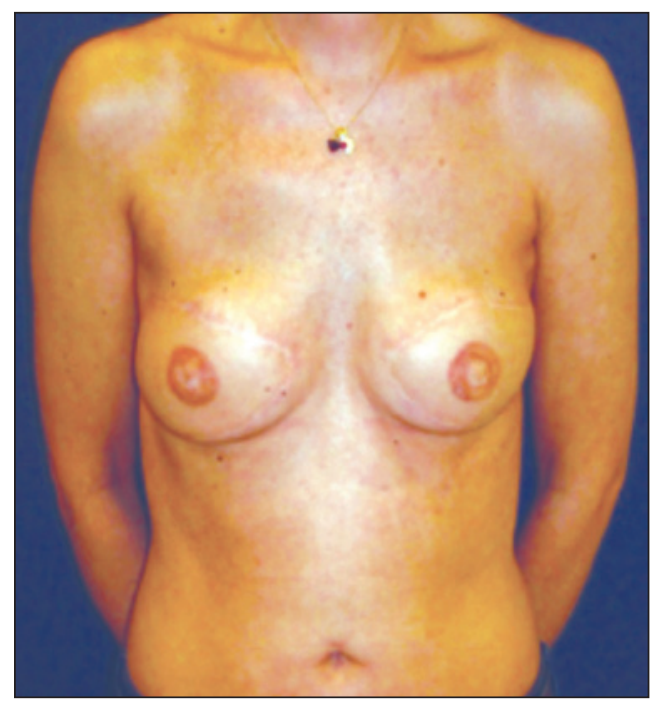

Figura $3 \mathrm{~A}$

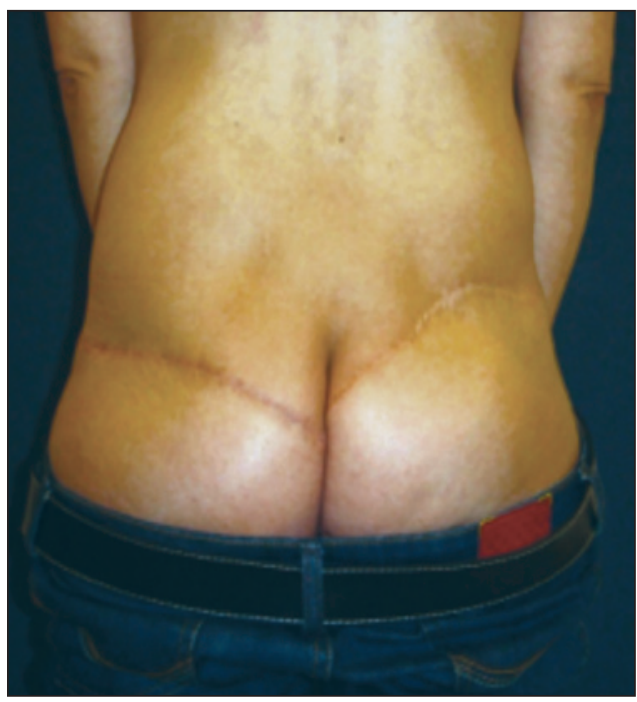

Figura 3B

Figuras 3A y 3B. Resultado al año tras reconstrucción mamaria bilateral con colgajo SGAP bilateral.

\section{- Otro tipo de colgajos}

Si el abdomen no proporciona una fuente adecuada de tejido para la reconstrucción de la mama, puede utilizarse tejido de los glúteos (colgajo SGAP/colgajo IGAP), de la cara interna de los muslos (colgajo TMG), de la zona lumbar (colgajo LAP), de la cara posterior del muslo (colgajo PAP) o incluso colgajo pediculado de la mama contralateral (colgajo "breast-sharing").

Estos colgajos no son comúnmente utilizados, y puede que no todos los centros ofrezcan estas técnicas. Estos colgajos suelen utilizarse cuando la paciente desea reconstrucción con tejido propio y no es posible realizar la reconstrucción con tejido abdominal, o con tejido de la región dorsal porque son personas muy delgadas o porque han tenido una cirugía previa que contraindica su uso. 


\section{- Lipomodelado o injerto de grasa (lipofilling)}

La técnica del injerto graso consiste en extraer la grasa sobrante de algunas zonas del cuerpo de la paciente mediante liposucción y procesarla para eliminar la sangre y otras substancias distintas de la grasa y, una vez purificada, transferirla a otras áreas mediante cánulas de infiltración para añadir volumen y así incrementar el tamaño, mejorar la forma y la textura y el perfil de las mamas.

Esta técnica permite conseguir buenos resultados en las reconstrucciones parciales de mama o al complementar otras técnicas reconstructivas (sobre todo las que emplean tejido propio de la paciente como el DIEP).

\section{Linfedema}

El linfedema se presenta en alrededor del $25 \%$ de pacientes afectas de cáncer de mama, afectando gravemente la calidad de vida de las pacientes ${ }^{(18)}$.

Para la mejoría del mismo han aparecido en lo últimos años diversas técnicas quirúrgicas, que pueden ser reconstructivas o paliativas.

En los casos de persistencia de funcionalidad del sistema linfático pueden realizarse técnicas reconstructivas como el trasplante autólogo de ganglios o las anastomosis linfático venosas. En casos muy avanzados la liposucción selectiva puede reducir el exceso de volumen y peso de la misma.

En casos de reconstrucción inmediata también puede realizarse la intervención para la prevención del linfedema, mediante la realización de un trasplante de ganglios tras el vaciamiento axilar, quedando así restituidas de una vez todas las alteraciones anatómicas ocasionadas por la mastectomía ${ }^{(19)}$.

\section{Discusión}

La reconstrucción mamaria muchas veces significa un paso primordial hacia la normalidad, no solo restituye un órgano perdido, sino que ayuda a recobrar la feminidad perdida y contribuye a olvidar la terrible lucha que se ha llevado contra la enfermedad. No interfiere ni en el tratamiento ni en el control de las pacientes, la prueba de ello es que cada día son más los oncólogos que recomiendan la reconstrucción mamaria ${ }^{(20)}$.

El objetivo del cirujano plástico es crear una nueva mama, lo más parecida a una mama natural. Para ello, a lo largo de los años, y a medida que han ido avanzando las técnicas quirúrgicas y los conocimientos anatómicos han ido apareciendo técnicas que permiten reconstrucciones de mayor calidad y más fisiológicas.

Lo ideal sería que todos los cirujanos plásticos que se dedican a la reconstrucción mamaria tuvieran un conocimiento y experiencia considerable sino en todas, en la mayoría de las técnicas reconstructivas. De las prótesis directas, pasando por los expansores o el dorsal ancho a las técnica más sofisticadas de colgajos microquirúrgicos de perforantes (DIEP, SIEA, SGAP ...), de todas ellas se debería tener un amplia experiencia para de esta manera poder elegir la técnica más apropiada o la combinación de ellas para cada paciente. Solo de esta se puede conseguir el resultado óptimo para poder mejorar la calidad de vida de nuestras pacientes. 


\section{- Lipomodelado o injerto de grasa (lipofilling)}

La técnica del injerto graso consiste en extraer la grasa sobrante de algunas zonas del cuerpo de la paciente mediante liposucción y procesarla para eliminar la sangre y otras substancias distintas de la grasa y, una vez purificada, transferirla a otras áreas mediante cánulas de infiltración para añadir volumen y así incrementar el tamaño, mejorar la forma y la textura y el perfil de las mamas.

Esta técnica permite conseguir buenos resultados en las reconstrucciones parciales de mama o al complementar otras técnicas reconstructivas (sobre todo las que emplean tejido propio de la paciente como el DIEP).

\section{Linfedema}

El linfedema se presenta en alrededor del $25 \%$ de pacientes afectas de cáncer de mama, afectando gravemente la calidad de vida de las pacientes ${ }^{(18)}$.

Para la mejoría del mismo han aparecido en lo últimos años diversas técnicas quirúrgicas, que pueden ser reconstructivas o paliativas.

En los casos de persistencia de funcionalidad del sistema linfático pueden realizarse técnicas reconstructivas como el trasplante autólogo de ganglios o las anastomosis linfático venosas. En casos muy avanzados la liposucción selectiva puede reducir el exceso de volumen y peso de la misma.

En casos de reconstrucción inmediata también puede realizarse la intervención para la prevención del linfedema, mediante la realización de un trasplante de ganglios tras el vaciamiento axilar, quedando así restituidas de una vez todas las alteraciones anatómicas ocasionadas por la mastectomía ${ }^{(19)}$.

\section{Discusión}

La reconstrucción mamaria muchas veces significa un paso primordial hacia la normalidad, no solo restituye un órgano perdido, sino que ayuda a recobrar la feminidad perdida y contribuye a olvidar la terrible lucha que se ha llevado contra la enfermedad. No interfiere ni en el tratamiento ni en el control de las pacientes, la prueba de ello es que cada día son más los oncólogos que recomiendan la reconstrucción mamaria ${ }^{(20)}$.

El objetivo del cirujano plástico es crear una nueva mama, lo más parecida a una mama natural. Para ello, a lo largo de los años, y a medida que han ido avanzando las técnicas quirúrgicas y los conocimientos anatómicos han ido apareciendo técnicas que permiten reconstrucciones de mayor calidad y más fisiológicas.

Lo ideal sería que todos los cirujanos plásticos que se dedican a la reconstrucción mamaria tuvieran un conocimiento y experiencia considerable sino en todas, en la mayoría de las técnicas reconstructivas. De las prótesis directas, pasando por los expansores o el dorsal ancho a las técnica más sofisticadas de colgajos microquirúrgicos de perforantes (DIEP, SIEA, SGAP ...), de todas ellas se debería tener un amplia experiencia para de esta manera poder elegir la técnica más apropiada o la combinación de ellas para cada paciente. Solo de esta se puede conseguir el resultado óptimo para poder mejorar la calidad de vida de nuestras pacientes. 
El objetivo de toda cirugía reparadora será el obtener el mejor resultado funcional y estético posible, con la menor morbilidad para el paciente.

La reconstrucción mamaria puede realizarse tanto de forma inmediata, como de forma diferida. No existe justificación oncológica para diferir la reconstrucción mamaria en las pacientes diagnosticadas de cáncer de mama ${ }^{(21)}$.

Durante las últimas dos décadas ha habido un incremento en la consideración de la reconstrucción mamaria inmediata. Previo a este periodo, las mujeres esperaban meses o incluso años a ser reconstruidas, debido a las preocupaciones de que la reconstrucción inmediata podría complicar las terapias adyuvantes o enmascarar una recurrencia. Sin embargo, los avances en los procedimientos quirúrgicos como una mayor evidencia acumulada que sugiere un bajo riesgo relacionado con la morbilidad de la patología en las pacientes que reciben la reconstrucción inmediata, han reforzado el rápido aumento de la reconstrucción en el momento de la mastectomía ${ }^{(22)}$.

La reconstrucción inmediata ofrece varias ventajas importantes sobre reconstrucción diferida, incluyendo la necesidad de una sola operación y la hospitalización, reducción de costos y un mejor resultado estético. Algunos autores han argumentado que la reconstrucción inmediata sirve como un amortiguador psicológico, pudiendo prevenir el trastorno psicológico y los problemas de adaptación que pueden ser observados en las primeras etapas después de la mastectomía. Si bien es cierto, que la literatura también ha descrito una mayor ansiedad en las pacientes en las que se realiza la reconstrucción inmediata coincidiendo con el diagnostico del cáncer de mama, por lo que el cirujano debe relacionarse con la paciente para adecuar el mejor momento reconstructivo ${ }^{(23)}$.

En el caso de pacientes que vayan a recibir radioterapia postoperatoria, la indicación de reconstrucción mamaria inmediata en pacientes con cáncer de mama es controvertida, por los posibles efectos negativos de ésta sobre la mama reconstruida. En principio, sería indicado no emplear reconstrucción con implantes (o mixta), por los efectos claramente negativos de la RT sobre la reconstrucción con los mismos. No hay acuerdo al respecto de la reconstrucción autóloga y a los efectos de la RT sobre ésta ${ }^{(24)}$.

Las pacientes tienen derecho a una valoración exhaustiva y rigurosa, por parte de su cirujano plástico, de la técnica más indicada para su intervención de reconstrucción mamaria, valoración que debe tener en cuenta el tratamiento oncológico que pueda estar recibiendo, como la radioterapia o la quimioterapia.

Por sus resultados y evolución posterior más naturales, la técnica con tejido autólogo debería emplearse, de hecho, como estándar en la reconstrucción mamaria, salvo en los casos en los que esté contraindicada, ya sea por las condiciones médicas de las pacientes o en las mujeres muy delgadas, con pocos tejidos para recrear la nueva mama, o en los que vaya en contra de las preferencias estéticas de las propias pacientes.

Pese a ser la opción preferible, la reconstrucción con tejido autólogo es la que menos se realiza en España por desinformación de las pacientes, carencias en las instalaciones y/o los materiales necesarios para su realización o inexistencia de Servicio de Cirugía Plástica en el centro hospitalario donde tiene lugar.

Como se ha comentado antes, el hecho de que sólo el hecho de que sólo el 30\% de las mujeres lleven a cabo la reconstrucción se debe, en parte, a una falta de información. Ésto puede afectar a la calidad de vida de las pacientes, por lo que sería imprescindible una implicación mayor para aportar información la información necesaria a las pacientes, por parte de todos los profesionales dedicados al tratamiento del cáncer de mama. 


\section{Conclusión}

La reconstrucción mamaria es un procedimiento electivo que tiene como objetivo final el mejorar la calidad de vida de las pacientes afectadas por cáncer de mama. De esta manera la elección de la técnica más apropiada se debe hacer de forma consensuada entre la paciente y su cirujano plástico. Para lograr la elección realmente adecuada de la técnica es fundamental que el cirujano plástico tenga la sensibilidad necesaria para entender a la paciente, la experiencia imprescindible sobre todas las técnicas y la capacidad de comunicación para transmitir a la paciente de una forma clara y honesta la información que le permita entender el procedimiento a realizar.

La reconstrucción mamaria ideal es aquella reconstrucción que aporta una nueva mama con las características de una mama natural. En definitiva que la mujer recupere una neo-mama que no solo parece una mama sino que la siente como tal, y todo ello con un resultado definitivo con la mínima morbilidad posible.

El cáncer de mama sigue siendo, aún en día, uno de los más frecuentes en las mujeres de nuestra sociedad. Para la mujer no es solo una terrible enfermedad sino que muy frecuentemente, como parte del tratamiento, conlleva la mastectomía o extirpación de la mama. Por tanto, la mujer, deberá enfrentarse al cáncer de mama y a la pérdida de un seno, que afectará a su calidad de vida.

Por tanto, la reconstrucción mamaria no debería considerarse un paso posterior, si no una parte imprescindible para un tratamiento integral.

\section{Referencias bibliográficas}

1. Asociación Española Contra el cáncer. 2016. Cáncer de mama. 2016. [Acceso 15 de agosto de 2017]. Disponible en: https://www.aecc.es/sobreelcancer/cancerporlocalizacion/ cancermama/paginas/incidencia.aspx

2. Al-Ghazal SK, Fallowfield L, Blamey RW. Does cosmetic outcome from treatment of primary breast cancer influence psychosocial morbidity? Eur J Surg Oncol 1999;25: 5713. doi: 10.1053/ejso.1999.0708

3. Contant CM, van Wercsh AME, Menke Pluymers MBE, et al. Satisfaction and prosthesis related complaints in women with immediate breast reconstruction following prophylactic and oncological mastectomy. Psychol Health Med 2004;9:71-85. doi:10.1080/135485003 10001637760

4. Helms RL, O'Hea EL, Corso M. Body image issues in women with breast cancer. Psychol Health Med 2008;13:313-25. doi: 10.1080/13548500701405509.

5. Montazeri A, Vahdaninia M, Harirchi I, Ebrahimi M, Khaleghi F, Jarvandi S. et al. Quality of life in patients with breast cancer before and after diagnosis: an eighteen months follow-up study. BMC Cancer 2008;8:330. doi: 10.1186/1471-2407-8-330.

6. Bellino S, Fenocchio M, Zizza M, Rocca G, Bogetti P, Bogetto F. Quality of life of patients who undergo breast reconstruction after mastectomy: effects of personality characteristics. Plast Reconstr Surg 2011;127:10-7. doi: 10.1097/PRS.0b013e3181f956c0

7. Roth RS, Lowery JC, Davis J, Wilkins EG. Psychological factors predict patients satisfaction with postmastectomy breast reconstruction. Plast Reconstr Surg 2007;119:2008-17. doi: 10.1097/01.prs.0000260584.09371.99

8. Reavey P, McCarthy CM. Update on breast reconstruction in breast cancer. Curr Opin Obstet Gynecol 2008;20:61-7. doi: 10.1097/GCO.0b013e3282f2329b. 
9. Estudio nacional de la Sociedad Española de Cirugía Plástica, Reparadora y Estética (SECPRE) sobre la calidad de tratamiento integral a las mujeres afectadas por cáncer de mama, octubre 2012. Depósito Legal M-33921-2012.

10. Pusic A, Thompson TA, Kerrigan CL, Sargeant R, Slezak S, Chang BW, et al.. Surgical options for the early-stage breast cancer: factors associated with patient choice and postoperative quality of life. Plast Reconstr Surg 1999;104:1325-33.

11. Cocquyt VF, Blondeel PN, Depypere HT, Van De Sijpe KA, Daems KK, Monstrey SJ, et al. Better cosmetic results and comparable quality of life after skin-sparing mastectomy and immediate autologous breast reconstruction compared to breast conservative treatment. Br J Plast Surg 2003;56:462-70. doi:10.1016/S0007-1226(03)00198-X

12. Dean C, Chetty U, Forrest AP. Effects of immediate breast reconstruction on psychosocial morbidity after mastectomy. Lancet 1983;1:459-62.

13. Masia J. Después del cáncer de mama: como mejorar la calidad de vida durante y después de la enfermedad. RBA Libros, 2009.

14. Elthair Y, Werners L, Dreise M, Zeijlmans van Emmichoven IA, Werker PM, de Bock GH. Which breast is the best? successful autologous or alloplastic breast reconstruction: patient-reported quality-of-life outcomes. Plast Reconstr Surg 2015;43:43-50. doi: 10.1097/PRS.0000000000000804.

15. Govshievich A, Somogyi RB, Brown MH. Conservative mastectomies and immediate reconstruction with the use of ADMs. Gland Surg 2015;4:453-62. doi: 10.3978/j. issn.2227-684X.2015.02.03

16. Skovsted Yde S, Brunbjerg ME, Damsgaard TE. Acellular dermal matrices in breast reconstructions - a literature review. J Plast Surg Hand Surg 2016;50:187-96. doi: 10.3109/2000656X.2016.1140053.]

17. Smith SL. Functional morbidity following latissimus dorsi flap breast reconstruction. J Adv Pract Oncol 2014;5:181-7.

18. DiSipio T, Rye S, Newman B, Hayes S. Incidence of unilateral arm lymphoedema after breast cancer: a systematic review and meta-analysis. Lancet Oncol 2013;14:500-15. doi: 10.1016/S1470-2045(13)70076-7.

19. Masia J, Pons G, Nardulli ML. Combined Surgical Treatment in Breast Cancer-Related Lymphedema. J Reconstr Microsurg 2016;32:16-27. doi: 10.1055/s-0035-1544182.

20. Veiga D, Ferreira L. Quality-of-life and self-esteem outcomes after oncoplastic breast-conserving surgery. Plast Reconstr Surg 2010;125:811-7. doi: 10.1097/ PRS.0b013e3181ccdac5.

21. Brennan ME, Spillane AJ. Uptake and predictors of post-mastectomy reconstruction in women with breast malignancy: Systematic review. Eur J Surg Oncol 2013;39:527-41. doi: 10.1016/j.ejso.2013.02.021.

22. Malata CM, McIntosh SA, Purushotham AD. Immediate breast reconstruction after mastectomy: A review. Br J Surg 2000:87:1455-72. doi: 10.1046/j.13652168.2000.01593.x

23. Roth RS, Lowery JC, Davis J, Wilkins E. Quality of life and affective distress in women seeking immediate versus delayed breast reconstruction after mastectomy for breast cancer. Plast Reconstr Surg 2005;16:993-1002

24. Kronowitz SJ, Hunt KK, Kuerer HM, Babiera G, McNeese MD, Buchholz TA. Delayedimmediate breast reconstruction. Plast Reconstr Surg 2004;113:1617-28. 